\title{
Evaluation of Whole Brain Health in Aging and Alzheimer's Disease: A Standard Procedure for Scoring an MRI-Based Brain Atrophy and Lesion Index
}

\author{
Hui Guo ${ }^{\mathrm{a}, \mathrm{b}}$, Xiaowei Song ${ }^{\mathrm{a}, \mathrm{c}, *}$, Matthias H. Schmidt ${ }^{\mathrm{d}, \mathrm{e}}$, Robert Vandorpe ${ }^{\mathrm{d}, \mathrm{e}}$, Zhan Yang ${ }^{\mathrm{a}, \mathrm{f}}$, \\ Emily LeBlanc $^{\mathrm{a}}$, Jing Zhang ${ }^{\mathrm{b}}$, Steven Beyea ${ }^{\mathrm{a}, \mathrm{d}, \mathrm{g}}$, Yunting Zhang ${ }^{\mathrm{b}}$ and Kenneth Rockwood ${ }^{\mathrm{c}, \mathrm{h}, *}$ \\ for the Alzheimer's Disease Neuroimaging Initiative ${ }^{1}$ \\ ${ }^{a}$ Neuroimaging Research Laboratory, Biomedical Translational Imaging Centre, QEII-IWK Health Sciences Centre \\ (the former National Research Council Canada's Institute for Biodiagnostics - Atlantic), Halifax, NS, Canada \\ ${ }^{\mathrm{b}}$ Department of Radiology, Tianjin Medical University General Hospital, Tianjin, China \\ ${ }^{\mathrm{c}}$ Department of Medicine, Dalhousie University, Halifax, NS, Canada \\ ${ }^{\mathrm{d}}$ Department of Radiology, Dalhousie University, Halifax, NS, Canada \\ ${ }^{\mathrm{e}}$ Department of Diagnostic Imaging, Capital District Health Authority, NS, Canada \\ ${ }^{\mathrm{f}}$ Department of Biology, Crandall University, Moncton, NB, Canada \\ ${ }^{\mathrm{g}}$ School of Health Sciences, Dalhousie University, Halifax, NS, Canada \\ ${ }^{\mathrm{h}}$ Centre for Healthcare of the Elderly, Capital District Health Authority, NS, Canada
}

\begin{abstract}
.
Background: The Brain Atrophy and Lesion Index (BALI), a semi-quantitative rating scale, has been developed to evaluate whole brain structural changes in aging and Alzheimer's disease (AD).

Objective: This study describes a standard procedure to score the BALI and train new raters for reliable BALI evaluation following this procedure.

Methods: Structural MRI of subjects in the Alzheimer's Disease Neuroimaging Initiative dataset who had 3.0T, T1, and T2 weighted MRI scans at baseline and at 6,12, and 24 month follow-ups were retrieved $(n=122$, including $24 \mathrm{AD}, 51$ mild cognitive impairment patients, and 47 healthy control subjects). Images were evaluated by four raters following training with a step-by-step BALI process. Seven domains of structural brain changes were evaluated, and a total score was calculated as the sum of the sub-scores.

Results: New raters achieved $>90 \%$ accuracy after two weeks of training. Reliability was shown in both intra-rater correlation coefficients (ICC $\geq 0.92, p<0.001$ ) and inter-rater correlation coefficients (ICC $\geq 0.88, p<0.001$ ). Mean BALI total scores differed by diagnosis $(\mathrm{F} \geq 2.69, p \leq 0.049)$ and increased consistently over two years.

Conclusion: The BALI can be introduced using a standard procedure that allows new users to achieve highly reliable evaluation of structural brain changes. This can advance its potential as a robust method for assessing global brain health in aging, AD, and mild cognitive impairment.
\end{abstract}

Keywords: Aging, Alzheimer's disease, brain, Brain Atrophy and Lesion Index (BALI), cognition, structural MRI

\footnotetext{
${ }^{*}$ Correspondence to: Xiaowei Song, PhD, MSCS, Neuroimaging Research Laboratory, Biomedical Translational Imaging Centre, Suite 3900, 1796 Summer Street, Halifax, Nova Scotia B3H 3A7, Canada. Tel.: +1 902473 1876; Fax: +1 902473 1050; E-mail: Xiaowei.Song@dal.ca; Kenneth Rockwood, MD, FRCPC, FRCP, Division of Geriatric Medicine, QEII Health Sciences Centre, Suite 1421-5955 Veterans Memorial Lane, Halifax, Nova Scotia, B3H 2E1, Canada. Tel.: +1 902473 8631; Fax: +1 902473 1050; E-mail: Kenneth.Rockwood@dal.ca.
}

\begin{abstract}
${ }^{1}$ Data used in preparation of this article were obtained from the Alzheimer's Disease Neuroimaging Initiative (ADNI) database (http://adni.loni.usc.edu). As such, the investigators within the ADNI contributed to the design and implementation of ADNI and/or provided data but did not participate in analysis or writing of this report. A complete listing of ADNI investigators can be found at: http://adni.loni.usc.edu/wp-content/uploads/how_to_ apply/ADNI_Acknowledgement_List.pdf. This paper obtained ADNI approval in January 2014.
\end{abstract}




\section{INTRODUCTION}

Multiple structural brain changes, such as global atrophy and white matter lesions, are common in older adults; they are more common still, and more severe, in people with dementia [1-4]. Importantly, brain changes can interact with each other and have additive impact on cognition [5-10]. To facilitate the evaluation of whole brain health in aging and dementia, taking into account multiple structural changes, a magnetic resonance imaging (MRI)-based semi-quantitative whole brain rating scale, i.e., the Brain Atrophy and Lesion Index (BALI), has been developed [11, 12]. The BALI evaluates several common structural changes in the aging brain in combination, by integrating several existing rating schema that focus on more specific/focal brain changes [13-16]. These changes include gray matter lesions (e.g., cortical infarcts) and subcortical dilated perivascular spaces (GM-SV), deep white matter lesions (DWM), periventricular white matter lesions (PV), lesions in the basal ganglia and surrounding areas (BG), lesions in the infratentorial compartment (IT), and global atrophy (GA). An "other findings" category records other possible changes (e.g., neoplasm, trauma, and malformations) [11, 12]. In contrast to volumetric measurements (of individual structures) that can be time consuming and/or require specialized expertise, the BALI provides a quicker alternative, permitting the evaluation of global brain health. The BALI has been shown to be reliable with content, criteria, and predictive validity $[17,18]$. These features make it promising in both clinical and research settings.

BALI has been validated based on either T1 or T2 weighted MRI (T1WI and T2WI), and for use with both high field (3.0T) and conventional (1.5T) systems $[17,18]$. Earlier studies demonstrate high correlation of the BALI scores with age and global cognitive testing scores; the BALI differs significantly among people with mild Alzheimer's disease (AD)/mild cognitive impairment (MCI) and cognitively healthy aging, and is associated with $\mathrm{AD}$ biomarkers [11, 12, 17]. Changes in BALI allow modeling of the dynamics of brain aging: despite an average decline, stabilization is common and sometimes improvement can be seen [19], providing a perspective on the interplay between harmful and protective factors in relation to brain health.

To date, BALI scoring has been performed solely by experienced neuroradiologists. As a standard procedure for brain image evaluation using BALI has not been defined, it is unknown whether new learners, novel to the method, can rate BALI. To improve its accessibility, tests on whether other qualified researchers, e.g., researchers with basic knowledge of human brain anatomy and MRI, can master the method with proper training are needed. Here, we report a standard procedure to enable robust structural MRI evaluation. Our primary objective is to describe the step-by-step process of BALI scoring, using either T1WI or T2WI (each is typically acquired in neuroimaging datasets). We also examine how well the BALI rating can be performed by raters who are new to the method following training, and address the most error-prone aspects in executing T1WI and T2WI evaluation by providing examples and practical solutions.

\section{MATERIALS AND METHODS}

\section{Data}

Data used in the preparation of this article were obtained from the Alzheimer's Disease Neuroimaging Initiative (ADNI) database (http://adni.loni.usc.edu) [20] with permission. The ADNI was launched in 2003 by the National Institute on Aging (NIA), the National Institute of Biomedical Imaging and Bioengineering (NIBIB), the Food and Drug Administration (FDA), private pharmaceutical companies and non-profit organizations, as a $\$ 60$ million, 5-year, public-private partnership. The primary goal of ADNI has been to test whether serial MRI, positron emission tomography, other biological markers, and clinical and neuropsychological assessment can be combined to measure the progression of MCI and early AD. Determination of sensitive and specific markers of very early AD progression is intended to aid researchers and clinicians to develop new treatments and monitor their effectiveness, as well as lessen the time and cost of clinical trials. The Principal Investigator of this initiative is Michael W. Weiner, MD, VA Medical Center and University of California - San Francisco. ADNI is the result of efforts of many co-investigators from a broad range of academic institutions and private corporations, and subjects have been recruited from over 50 sites across the U.S. and Canada. The initial goal of ADNI was to recruit 800 subjects but ADNI has been followed by ADNI-GO and ADNI-2. To date these three protocols have recruited over 1500 adults, ages 55 to 90 , to participate in the research, consisting of cognitively normal older individuals, people with early or late MCI, and people with early AD. The follow up duration of each group is specified in the protocols for ADNI-1, ADNI-2, and ADNI-GO. Subjects originally recruited for ADNI-1 and ADNI-GO had the option 
Table 1

Baseline demographic and clinical characteristics, by diagnosis

\begin{tabular}{|c|c|c|c|c|c|c|}
\hline \multirow[t]{2}{*}{ Group } & \multirow[t]{2}{*}{$\mathrm{AD}$} & \multicolumn{2}{|c|}{ MCI } & \multirow[t]{2}{*}{$\mathrm{HC}$} & \multirow[t]{2}{*}{$\mathrm{K}-\mathrm{W} / \chi^{2}$} & \multirow[t]{2}{*}{$p$} \\
\hline & & Conversion & Stable & & & \\
\hline Sample size & 24 & $25^{\mathrm{a}}$ & 26 & 47 & & \\
\hline Female $(\%)$ & 58.3 & 28.0 & 42.3 & 59.6 & 7.82 & 0.050 \\
\hline Education (y) & $14.1 \pm 3.2$ & $15.1 \pm 3.9$ & $16.4 \pm 2.6$ & $16.5 \pm 2.2$ & 11.07 & 0.011 \\
\hline Age $(y)$ & $74.3 \pm 9.0$ & $74.4 \pm 8.4$ & $74.0 \pm 5.9$ & $75.4 \pm 5.0$ & 0.95 & 0.815 \\
\hline $\operatorname{CDR}(0-3)^{\mathrm{b}}$ & $0.7 \pm 0.0(0.5)$ & $0.5 \pm 0.0(0.5)$ & $0.5 \pm 0.0(0.5)$ & $0.0 \pm 0.0(0.0)$ & 112.93 & $<0.001$ \\
\hline MMSE (/30) & $22.9 \pm 2.2$ & $26.2 \pm 1.7$ & $27.8 \pm 1.7$ & $29.5 \pm 0.7$ & 86.08 & $<0.001$ \\
\hline ADAS-cog $(/ 70)$ & $17.9 \pm 5.9$ & $14.0 \pm 3.1$ & $9.6 \pm 3.7$ & $4.9 \pm 2.3$ & 84.35 & $<0.001$ \\
\hline $\mathrm{A} \beta_{1-42}(\mathrm{pg} / \mathrm{ml})^{\mathrm{c}}$ & $147.8 \pm 43.4$ & $136.8 \pm 28.9$ & $176.3 \pm 57.2$ & $219.0 \pm 54.4$ & 21.43 & $<0.001$ \\
\hline Tau $(\mathrm{pg} / \mathrm{ml})^{\mathrm{c}}$ & $129.6 \pm 28.9$ & $93.8 \pm 35.5$ & $82.3 \pm 37.9$ & $72.5 \pm 31.7$ & 20.90 & $<0.001$ \\
\hline p-tau $(\mathrm{pg} / \mathrm{ml})^{\mathrm{c}}$ & $46.5 \pm 18.7$ & $33.3 \pm 13.8$ & $27.9 \pm 11.1$ & $26.8 \pm 14.1$ & 14.63 & 0.002 \\
\hline
\end{tabular}

Data are presented as mean \pm standard deviation, unless otherwise indicated. AD, Alzheimer's disease; MCI, Mild cognitive impairment; HC, healthy control; CDR, Clinical Dementia Rating; MMSE, Mini-Mental Status Examination; ADAS-cog, Alzheimer's Disease Assessment

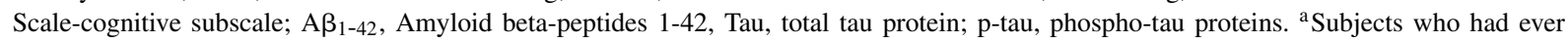
converted from MCI to AD during the 24-month follow-up ( $n=2$ at six months; $n=10$ at twelve months including one person who converted back to MCI at 24 months; $n=13$ at twenty-four months). ${ }^{b}$ Median in brackets. ${ }^{\mathrm{c}}$ Tested annually in a portion of this $\mathrm{ADNI}$ sample (AD $=15$, MCI conversion $=12, \mathrm{MCI}$ stable $=18, \mathrm{HC}=24) . \mathrm{K}-\mathrm{W} / \chi^{2}$, statistics for the Kruskal-Wallis nonparametric test or Chi-Square test. $p$ level of significance.

to be followed in ADNI-2. For up-to-date information, see http://www.adni-info.org. Participants who were randomized for 3.0T scans were first screened at $1.5 \mathrm{~T}$ for the inclusion/exclusion criteria [20-22].

For this study, T1WI and T2WI of all ADNI-1 subjects who had 3.0T scans at baseline and at 6 , 12, and 24-months were retrieved (Table 1; $n=122$; women $=49.2 \%$; mean age $=75.6 \pm 6.8$ years at baseline; $\mathrm{AD}=24, \mathrm{MCI}=51$, healthy controls $(\mathrm{HC})=47$ ). In addition to MRI data, clinical assessment data were obtained from the ADNI clinical dataset. These included the Mini-Mental State Examination, Clinical Dementia Rating scale, and the Alzheimer's Disease Assessment Scale - cognitive subscale. Diagnostic categorization, i.e., AD, MCI, and $\mathrm{HC}$, was made by ADNI site physicians in accordance with the National Institute of Neurologic and Communicative Disorders and Stroke/Alzheimer's Disease and Related Disorders Association (NINCDS/ADRDA) criteria and was reviewed by ADNI clinical monitors [22-24]. For the subjects with cerebrospinal fluid (CSF) biomarkers tests, the Amyloid beta 1-42 peptide $\left(\mathrm{A} \beta_{1-42}\right)$, phospho-tau and total tau protein data were obtained [25].

\section{Brain images}

Both T1WI and T2WI were evaluated. T1WI is commonly acquired in both clinical and research datasets for anatomy, tissue segmentation, and registration purposes. On T1WI, the CSF has low signal intensity (SI) and appears dark. Normal GM has lower SI than normal WM, and lesions typically have reduced SI, compared to background tissue, on T1WI. In this ADNI sample, the T1WI scans were 3D MP-RAGE $(\mathrm{TR} / \mathrm{TE}=2300-3000 / 3 \mathrm{~ms}$; flip angle $=8-9^{\circ}$; slice thickness $=1.2 \mathrm{~mm} ; 256$ reconstructed axial slices).

T2WI is also widely used, especially in clinical settings, to detect pathological changes. On T2WI, the CSF has high SI and appears bright. Normal GM has higher SI than normal WM. Lesions typically have increased SI, compared to background tissue, on T2WI. In this dataset, the T2WI scans were 2D FSE/TSE $(\mathrm{TR} / \mathrm{TE}=3000-4000 / 96-99 \mathrm{~ms}$; flip angle $=90$ or $150^{\circ}$; slice thickness $=3 \mathrm{~mm}$; 48 axial slices).

\section{Rating of Brain Atrophy and Lesion Index (BALI)}

The BALI is a semi-quantitative measurement that integrates information from multiple sources and is well suited to evaluating changes at the whole brain level. To produce a BALI total score, seven categories of brain changes are assessed based on their MRI appearance and localization (Fig. 1A; Fig. 1B).

Category 1. Gray matter lesions and subcortical dilated perivascular spaces (GM-SV). This category includes lesions located in the GM of the cerebral cortex (e.g., foci of encephalomalacia due to old infarcts) and juxtacortical dilated perivascular spaces located within $4 \mathrm{~mm}$ of the corticomedullary junction [3, 26-29].

Category 2. Deep white matter lesions (DWM). This category includes lesions located between the juxtacortical and periventricular white matter. These 


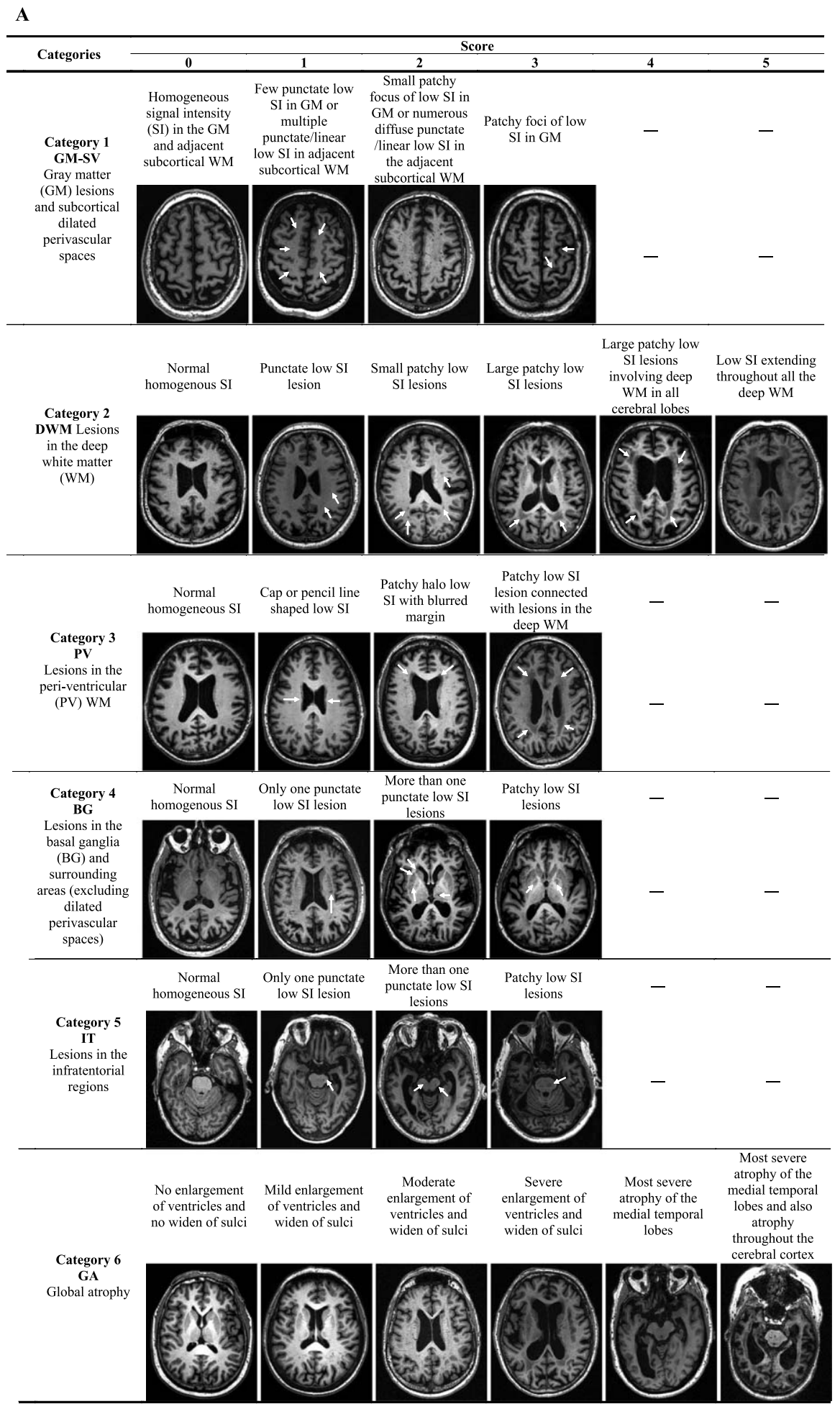

Fig. 1A. Evaluation of the Brain Atrophy and Lesion Index (BALI) based on T1WI. 
B

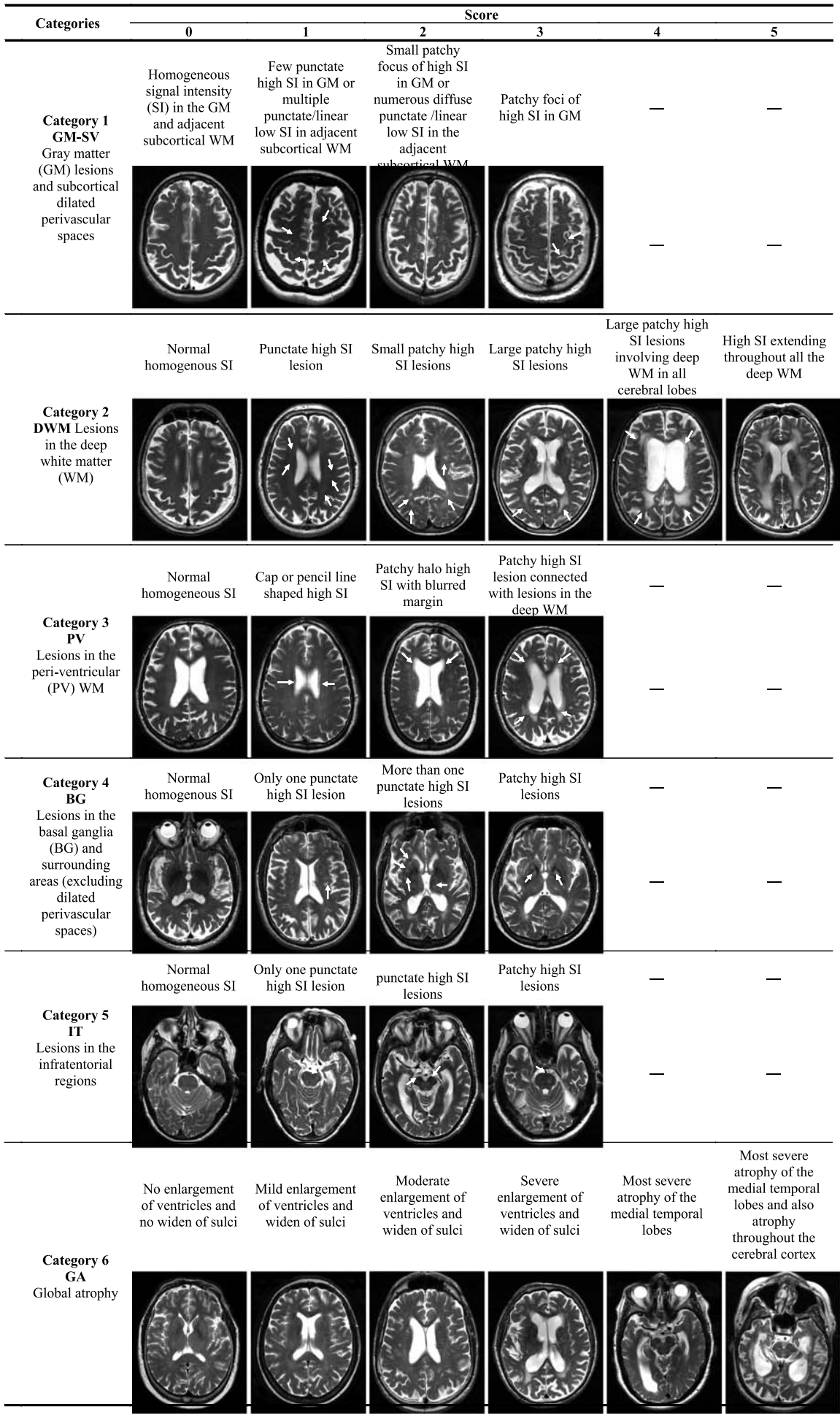

Fig. 1B. Evaluation of the Brain Atrophy and Lesion Index (BALI) based on T2WI. 
most commonly reflect chronic small vessel ischemic changes [3, 30].

Category 3. Periventricular white matter lesions (PV). This category includes lesions in the WM, contiguous with the margins of the lateral ventricles and located within $13 \mathrm{~mm}$ of the ventricular surface [3]. These most commonly reflect chronic small vessel ischemic changes.

Category 4. Lesions in the basal ganglia and surrounding areas $(\mathrm{BG})$. This category includes the caudate nucleus, putamen, globus pallidus, internal capsule, thalamus, external capsule, claustrum and insular lobes. Lesions here most commonly reflect lacunar infarcts. Dilated perivascular spaces at the ventral aspect of the lentiform nuclei were excluded, given that they are common even in healthy younger adults [31].

Category 5. Lesions in the infratentorial compartment (IT) including in the cerebellum and the brain stem (the medulla oblongata, pons, and midbrain). Such lesions typically are due to chronic small vessel ischemic changes and lacunar infarcts.

Category 6. Global atrophy (GA). This category assesses the shrinkage of the whole brain and the enlargement of the ventricles and sulci. The highest score is given for atrophy that is most severe in the mesial temporal lobes.

Category 7. Other findings. These include other changes such as neoplasm, trauma, normal pressure hydrocephalus, and malformations. (Note that no category 7 lesion was observed in the present dataset.)

A value between $0-3$ is assigned to each category, depending on the severity of change, with a higher score denoting greater severity. Values of 4 and 5 are used to capture more severe changes in the DWM and GA categories. When a lesion is intermediate in severity, the lower of the two possible scores is assigned. The BALI total score is then calculated as the sum of the sub-scores of all seven categories (maximum $=25$, higher score denoting more severe lesions and/or more categories of lesions; Fig. 1A; Fig. 1B).

In this study, the images were evaluated by four researchers, including one neuroradiologist with nine years of experience of brain MRI evaluation (Rater \#1). Other raters included a $\mathrm{PhD}$ neuroimaging scientist who was familiar with the method (Rater \#2), a $\mathrm{PhD}$ biologist who took part in ageing research (Rater \#3), and a graduate student working in dementia neuroimaging (Rater \#4). The latter two raters were new to BALI and obtained training with BALI prior to participating in the study. Two additional experienced neuroradiologists familiar with BALI assisted with the training and were available for questions arising during the scoring process.

During rating, images were displayed using the MRIcron software package on a 17-inch computer screen with $1920 \times 1080$ resolution, and three times magnification. Rating was performed on axial images, with scores in each category assigned in the order shown in Fig. 1A; Fig. 1B. Raters were blinded with respect to the subject's demographics, diagnosis, cognitive testing scores, and scan time. T1WI and T2WI of each subject were assessed separately on different days in random order to minimize recall bias.

\section{Training}

A working knowledge of human neuroanatomy and structural MRI is a prerequisite for a new rater [32, 33]. In the present study, the training phase began with study of the BALI rating schema (Fig. 1A; Fig. 1B), including text descriptions and image illustrations for both T1WI (Fig. 1A) and T2WI (Fig. 1B). Trainees were given the opportunity to observe BALI evaluations made by neuroradiologists, as well as the opportunity to practice BALI scoring with expert feedback. This process was completed in an average of 20 working hours. Then, the new raters were assigned 20 training image datasets to evaluate every day without immediate feedback. Their scores were compared daily to those of the neuroradiologists, at which time feedback was provided (Fig. 2). This process continued until $\geq 90 \%$ agreement with the neurologists was achieved on three consecutive days. Thereafter, each rater was given all of the image datasets and allowed to rate them independently, completely blinded to other raters' scores. Consultations with the neuroradiologists other than for the training images were permitted only concerning the nature of a change, not concerning how such change should be scored with BALI.

\section{Analysis}

Intra and inter-rater agreement rates were assessed for BALI total scores using the intraclass correlation coefficient (ICC), applying a two-way (sample, rater) random model, and examined using $100 \%$ subjects, and $50 \%, 20 \%, 10 \%$ random subsampling. To determine intra-rater reliability, BALI rating on T2WI was repeated using $10 \%$ random sub-sample following the same schema.

Demographic characteristics across diagnostic groups were examined using the Kruskal-Wallis nonparametric test for interval data (e.g., age) and 


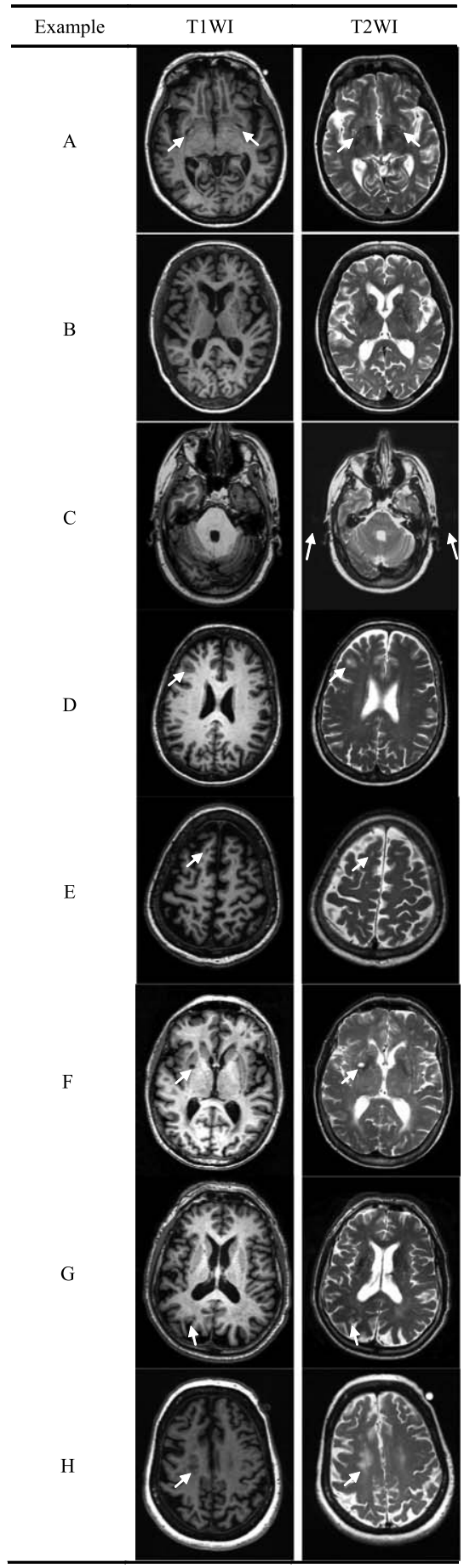

Fig. 2. Common errors made by learners of the BALI rating (white arrows). A) Over-scoring of dilated perivascular spaces along the lenticulostriate arteries entering the basal ganglia through the anterior perforated substance. B) Over-scoring ventricular enlargement (especially on T2WI) as periventricular lesion. C) Over-scoring in the infratentorial region as large confluent lesions on T2WI, due to incorrect evaluation of flow artifact (evident on the background). D) Mis-scoring normal sulci structures as gray matter lesions, due to incorrect evaluation of the partial volume effect of cerebral spinal fluid on T2WI. E) Mis-scoring subcortical white matter lesions as subcortical dilated perivascular spaces. F) Under-scoring lacunes located in the basal ganglia region (incorrectly evaluated as dilated perivascular spaces). G) Under-scoring gray matter lesions especially on T1WI. H) Under-scoring the periventricular white matter (WM) lesions located in the area superior to the ventricle that are connected with lesions in the deep WM. 
Table 2

Interrater agreement rates for the Brain Atrophy and Lesion Index (BALI) total scores, using different sub-samples

\begin{tabular}{|c|c|c|c|c|c|c|c|c|c|}
\hline & \multirow[t]{2}{*}{ Image type } & \multicolumn{2}{|c|}{$100 \%$ sample } & \multicolumn{2}{|c|}{$50 \%$ sub-sample } & \multicolumn{2}{|c|}{$20 \%$ sub-sample } & \multicolumn{2}{|c|}{$10 \%$ sub-sample } \\
\hline & & ICC & $95 \% \mathrm{CI}$ & ICC & $95 \% \mathrm{CI}$ & ICC & $95 \% \mathrm{CI}$ & ICC & $95 \% \mathrm{CI}$ \\
\hline \multirow[t]{2}{*}{ All raters } & T1WI & 0.92 & $0.91-0.93$ & 0.92 & $0.91-0.94$ & 0.92 & $0.90-0.94$ & 0.95 & $0.92-0.97$ \\
\hline & $\mathrm{T} 2 \mathrm{WI}$ & 0.91 & $0.90-0.93$ & 0.91 & $0.89-0.93$ & 0.92 & $0.89-0.94$ & 0.93 & $0.90-0.96$ \\
\hline Raters 1 & T1WI & 0.93 & $0.92-0.94$ & 0.94 & $0.92-0.95$ & 0.95 & $0.93-0.97$ & 0.96 & $0.93-0.98$ \\
\hline versus 2 & T2WI & 0.92 & $0.91-0.94$ & 0.93 & $0.91-0.94$ & 0.93 & $0.90-0.95$ & 0.95 & $0.91-0.97$ \\
\hline Raters 1 & T1WI & 0.94 & $0.93-0.95$ & 0.95 & $0.93-0.96$ & 0.94 & $0.91-0.96$ & 0.95 & $0.91-0.97$ \\
\hline versus 3 & T2WI & 0.93 & $0.92-0.95$ & 0.93 & $0.92-0.95$ & 0.93 & $0.90-0.95$ & 0.93 & $0.88-0.96$ \\
\hline Raters 1 & T1WI & 0.95 & $0.94-0.96$ & 0.95 & $0.93-0.96$ & 0.95 & $0.93-0.97$ & 0.96 & $0.94-0.98$ \\
\hline versus 4 & T2WI & 0.93 & $0.91-0.94$ & 0.92 & $0.90-0.94$ & 0.93 & $0.90-0.95$ & 0.95 & $0.92-0.97$ \\
\hline Raters 2 & T1WI & 0.89 & $0.87-0.91$ & 0.88 & $0.85-0.91$ & 0.88 & $0.83-0.92$ & 0.92 & $0.87-0.96$ \\
\hline versus 3 & T2WI & 0.90 & $0.88-0.92$ & 0.89 & $0.86-0.91$ & 0.90 & $0.85-0.93$ & 0.91 & $0.84-0.95$ \\
\hline Raters 2 & T1WI & 0.91 & $0.90-0.93$ & 0.92 & $0.89-0.94$ & 0.91 & $0.88-.094$ & 0.95 & $0.91-0.97$ \\
\hline versus 4 & T2WI & 0.90 & $0.88-0.92$ & 0.91 & $0.88-0.93$ & 0.91 & $0.86-0.94$ & 0.94 & $0.89-0.96$ \\
\hline Raters 3 & T1WI & 0.92 & $0.90-0.93$ & 0.92 & $0.89-0.93$ & 0.90 & $0.86-0.93$ & 0.93 & $0.87-0.96$ \\
\hline versus 4 & T2WI & 0.90 & $0.88-0.91$ & 0.89 & $0.86-0.91$ & 0.90 & $0.86-0.93$ & 0.92 & $0.86-0.95$ \\
\hline
\end{tabular}

Intraclass correlation coefficients (ICC) analysis for the BALI total scores. CI, confidence interval. Level of significance $p<0.001$ for all.

Chi-Square for categorical data (e.g., gender). Comparisons of the mean values of the BALI total scores and the sub-scores by time and/or diagnostic groups were conducted using ANOVA and the Kruskal-Wallis nonparametric tests respectively. Pair-wise multiple comparisons were tested for least significant difference (LSD). The interrelations of the BALI total scores between T1WI and T2WI at different visit times were examined using correlation analyses. All analyses were performed using PASW 17 and codes developed using Matlab 2013. The level of significance was set at $p<0.05$.

\section{RESULTS}

Each rater completed the evaluation of the entire dataset (i.e., 122 subjects, 4 time-points, 2 image types; Supplementary Table 1). Evaluating one image set, i.e., either T1WI or T2WI of a subject, typically took 4-8 minutes to complete, and seldom exceeded $10 \mathrm{~min}-$ utes. High agreement rates were observed for the total score (intra-rater: ICC $\geq 0.92,95 \%$ confidence interval $(\mathrm{CI})=0.81-0.96, p<0.001$; inter-rater: $\mathrm{ICC} \geq 0.91$, $95 \% \mathrm{CI}=0.90-0.94, p<0.001$ between the less experienced raters and the neuroradiologist rater; $\mathrm{ICC} \geq 0.88$, $95 \% \mathrm{CI}=0.85-0.91, p<0.001$ between the less experienced raters themselves; Table 2). No statistical difference was found for the rater-based subscores (Supplementary Table 1).

The mean BALI total scores differed significantly among diagnostic groups, with AD group showing the greatest scores, and $\mathrm{HC}$ and MCI-stable groups showing the lowest, which differed significantly even at baseline ( $\mathrm{F} \geq 2.69, p \leq 0.049$; Supplementary Table 1;
Fig. 3). The GA subscore differed significantly by diagnosis, whereas other subscores tended to increase with time from baseline, and in subjects with AD (Supplementary Table 1). An increase in the mean value of the BALI scores with time (as people aged) was observed in each diagnostic group, consistent across the raters. At 12 months, MCI converters had a similar BALI mean score as did people with $\mathrm{AD}$ at baseline (all-rater mean BALI total $11.8 \pm 2.7$ versus $11.8 \pm 2.4$ for T1WI, $12.5 \pm 2.7$ versus $12.3 \pm 2.2$ for T2WI; ANOVA LSD $|t| \leq 0.08, p \geq 0.936$; Fig. 3). The BALI total score significantly correlated with age and the global cognitive testing scores (Supplementary Table 2). All subscores were associated with age, while many of the subscores (especially GA, but not IT and GM-SV) were also associated with cognition (Supplementary Table 2).

These observations held true using both T1WI and T2WI images (Table 3; Supplementary Table 1). The T1WI and T2WI images were highly correlated across assessment time and rater, with the T2W1 scores being slightly higher than T1WI $(r \geq 0.91, p<0.001)$.

\section{DISCUSSION}

This study describes a step-by-step procedure to score the BALI, a global evaluation of several common changes in the aging brain, based on T1WI or T2WI. Our data showed that researchers with a basic understanding of human anatomy and MRI were able to master the method, following training in lesion identification and scoring with the standard procedure provided here. Each rater independently evaluated the images resulting in both high intra-rater consis- 

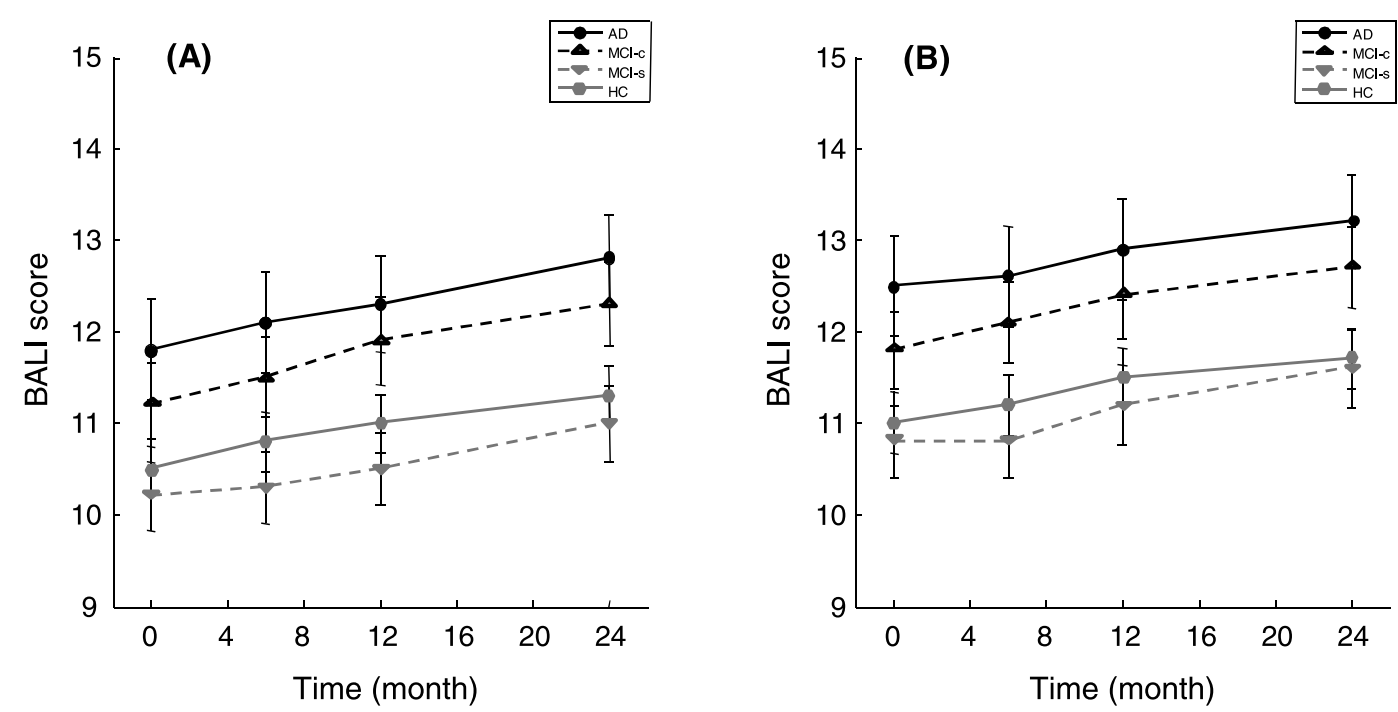

Fig. 3. The mean Brain Atrophy and Lesion Index (BALI) score at each assessment time. A) Based on T1WI. B) Based on T2WI. Data presented are the group means of all raters and the standard errors (at each time point $n=122$ of the same subjects). AD, Alzheimer's disease; MCI-c, Mild cognitive impairment converted to AD; MCI-s: stable MCI; HC, healthy control subjects.

Table 3

Correlations between T1WI and T2WI based Brain Atrophy and Lesion Index (BALI) total scores

\begin{tabular}{|c|c|c|c|c|c|c|c|c|c|}
\hline & \multirow[t]{2}{*}{ Image type } & \multicolumn{2}{|c|}{ Baseline } & \multicolumn{2}{|c|}{6 months } & \multicolumn{2}{|c|}{12 months } & \multicolumn{2}{|c|}{24 months } \\
\hline & & BALI & $\mathrm{r}$ & BALI & $\mathrm{r}$ & BALI & $\mathrm{r}$ & BALI & $\mathrm{r}$ \\
\hline \multirow[t]{2}{*}{ Rater 1} & T1WI & $10.7 \pm 2.4$ & 0.95 & $11.0 \pm 2.4$ & 0.96 & $11.3 \pm 2.4$ & 0.94 & $11.8 \pm 2.4$ & 0.94 \\
\hline & T2WI & $11.3 \pm 2.5$ & & $11.5 \pm 2.4$ & & $11.9 \pm 2.4$ & & $12.2 \pm 2.4$ & \\
\hline \multirow[t]{2}{*}{ Rater 2} & T1WI & $10.8 \pm 2.5$ & 0.93 & $11.1 \pm 2.5$ & 0.93 & $11.2 \pm 2.5$ & 0.94 & $11.6 \pm 2.5$ & 0.96 \\
\hline & T2WI & $11.2 \pm 2.5$ & & $11.5 \pm 2.4$ & & $11.8 \pm 2.5$ & & $12.2 \pm 2.5$ & \\
\hline \multirow{2}{*}{ Rater 3} & T1WI & $10.8 \pm 2.2$ & 0.93 & $11.0 \pm 2.2$ & 0.92 & $11.2 \pm 2.2$ & 0.92 & $11.7 \pm 2.1$ & 0.93 \\
\hline & $\mathrm{T} 2 \mathrm{WI}$ & $11.3 \pm 2.2$ & & $11.5 \pm 2.2$ & & $11.7 \pm 2.2$ & & $12.2 \pm 2.1$ & \\
\hline \multirow[t]{2}{*}{ Rater 4} & T1WI & $10.8 \pm 2.3$ & 0.93 & $11.1 \pm 2.3$ & 0.96 & $11.4 \pm 2.4$ & 0.96 & $11.8 \pm 2.2$ & 0.97 \\
\hline & T2WI & $11.5 \pm 2.3$ & & $11.6 \pm 2.4$ & & $11.9 \pm 2.5$ & & $12.1 \pm 2.4$ & \\
\hline
\end{tabular}

Values for BALI are presented as mean \pm standard deviation. r: correlation coefficient. Level of significance $p<0.001$ for all.

tency and high inter-rater agreement rates. The BALI scores given by each rater differed by diagnosis and strongly related to the clinical assessments, to which they were blinded. The BALI scores were correlated with age and global cognitive assessments, and consistently reflected the trend of average decline of whole brain health over time and MCI to AD conversion. Even though such associations were often also found for changes in the individual BALI categories, combining them in the summary score allowed evaluation of their overall impact on brain aging. In short, our study suggests that MRI-based BALI can serve as a robust method for evaluating whole brain structural changes in relation to age, cognition and $\mathrm{AD}$ dementia. The data support the involvement of qualified nonneuroradiologists to score BALI after proper training, suggesting the potential for widespread usefulness of this tool.
As might be expected, less experienced raters occasionally expressed uncertainty and were occasionally found to have made mistakes upon consultation with a neuroradiologist. The following eight errors were most common (Fig. 2A-H): 1) Figure 2A: Over-scoring for dilated perivascular spaces when evaluating the BG category. Given the high prevalence of the dilated perivascular spaces in this region in normal persons at all ages, they should not be counted as lesions (unlike foci of chronic small vessel ischemic change, gliosis or demyelination). Raters should be able to differentiate dilated perivascular spaces based on their location at the ventral aspect of the lentiform nuclei, ovoid shape, and CSF signal intensity; 2) Figure 2B: Over-scoring of global atrophy on T2WI. CSF is emphasized on T2WI, and atrophy appears more severe, compared to TIWI. This pitfall is avoided by consistently following the examples of GA rating provided in the BALI 
atlas (Fig. 1); 3) Figure 2C: Misinterpretation of artifacts as lesions, especially on T2WI in the IT category. Adjusting image brightness can help detect the artifact on the background; 4) Figure 2D: Misinterpretation of the partial volume effect around sulci or ventricles. Tracing structures on consecutive images by scrolling up and down can help distinguish a lesion from a partial volume effect of CSF; 5) Figure 2E: Misinterpretation of subcortical WM lesions as GM lesions. Careful assessment of the location of a lesion with respect to the GM-WM junction can prevent this error. It may help to scroll up and down to localize the lesion with respect to the GM-WM junction; 6) Figure 2F: Misinterpreting lacunar infarcts in the basal ganglia as dilated perivascular spaces when rating the BG category. Lacunes are more often asymmetrical and have an irregular outline, with marginal gliosis on T2WI; 7) Figure 2G: Underscoring of grey matter lesions especially on T1WI. This can be avoided by systematically tracing the cortical ribbon; 8) Figure 2H: Under-scoring periventricular lesions extending to the deep WM, especially superior to the lateral ventricles. The contiguity of WM lesions with the superior margins of the lateral ventricles can be difficult to appreciate on axial images. This pitfall can be avoided by tracing the margins of WM lesions carefully and scrolling the images up and down to establish contiguity with the ventricle.

Our study has some limitations. Neuroradiological expertise is critical during the training phase. Inexperienced raters will occasionally encounter unusual cases where the anatomy, the pathology, or both are difficult to interpret. In such cases, neuroradiological consultation is still necessary for clarification about lesion identification. Here, an important challenge was that only axial images were available in the T2WI dataset. Comparisons on the imaging types prevented them from cross-correlating lesions on multiple planes, as often occurs in clinical radiology. The scores were typically higher on T2WI than on T1WI. This is explained by the fact that most lesions are more conspicuous on T2WI. Occasionally, images fall between two BALI scores, and a judgment must be made with respect to scoring. In such cases, a consistent approach should be taken by all raters involved. In our study, every rater was told to assign the lower one of the two possible scores when there was equipoise between the two scores. This approach is recommended in further research aimed at validating the method. Gray matter lesions were observed relatively less often and their correlations with dilated perivascular spaces have been reported [28, 29]. The juxtacortical white matter and gray matter also have similar vascular supply and can be susceptible to the same ischemic disease [3]. Given that lesions involving either or both gray matter lesions and dilated perivascular spaces can have a similar morphological appearance that might not always be easily distinguished on T1WI and T2WI, they were scored in the same category. Further investigations are needed to understand better their pathological importance. The judgment on lesion size (e.g., "large confluent lesions") may differ for different categories (e.g., DWM versus BG and IT). In this regard, measurement, e.g., in $\mathrm{mm}$, may help, as long as precision and simplicity are balanced. In addition, distributions of lesions in different lobes can be associated with different cognitive domains and differ by diagnosis [34-37]. How to distinguish these warrants further research focusing on differential diagnosis using BALI.

In spite of these limitations, BALI has several strengths. It has demonstrated content, criteria and predictive validity, and takes only a few minutes to perform, thus offering a relatively efficient way to semi-quantitatively evaluate whole brain health in aging. It is based on structural MRI, which has been an integral part and standard component of the clinical AD assessment of AD dementia [38]. Although recent developments may allow function-based protocols, their clinical roles are yet to be established. BALI scoring is also less affected by imaging parameters and noise. This is an advantage for multicenter trials in which MRI scanners, scan parameters, and image quality often vary from site to site, despite the increasingly reorganized call for standardization of imaging data acquisition and processing [39, 40]. Further, some lesions that cannot be accurately quantified by automated analyses (e.g., segmentation of the basal ganglia regions relies on tiresome manual input due to tissue SI variability), are not a concern with BALI scoring.

Furthermore, the BALI score takes into account both significant and clinically apparent changes, as well as early subtle changes that are seemingly clinically silent. With the advances of high-field MRI technology, subtle imaging changes become more relevant, as they may be indicative of substantial underlying pathology at the histological level. The neuropathological basis of MR findings in aging and dementia can be complex, with multiple processes converging on similar structural changes to mediate cognitive decline [30, 34, 35, 41, 42]. For example, atrophy can emerge from the accumulation of pathological deficits, including loss of synapses, dendritic processes, and neurons [43, 44]. White matter lesions can also involve multiple causes (cerebral ischemia, demyelination, microcystic infarcts, venous collageno- 
sis, and gliosis) [3, 43, 45]. These may collectively affect cognition, even though individual changes sometimes show little impact. BALI permits evaluation of the collective effect of such changes.

In conclusion, structural brain changes with aging are common, and can be of uncertain importance. In general, individually small effects can summarize to be associated with clinically detectable impacts. The BALI is a straightforward means of summarizing these many changes. In this study, a standard procedure of scoring the BALI was tested for systematic evaluation of whole brain health in aging and $\mathrm{AD}$, and demonstrated high reliability for training new raters. Here, the well-established ADNI dataset was used to validate this process. Further research will be needed for validation with other datasets. This research can allow a better uptake of whole brain health evaluation in aging and AD.

\section{ACKNOWLEDGMENTS}

This research was supported by a Catalyst Grant on Secondary Neuroimaging Analysis from the Canadian Institutes of Health Research (CSE125739) and a China-Canada Joint Health Research Project from the National Science Foundation of China and the Canadian Institutes of Health Research (NSFCIHR81010072). Additional funding for analysis came from the Fountain Innovation Fund of the Queen Elizabeth II Health Sciences Research Foundation, Halifax, Canada. HG was supported by a collaborative project between the National Research Council Canada's Institute for Biodiagnostics - Atlantic and the General Hospital of Tianjin Medical University as a visiting postdoctoral research fellow to work in Halifax. KR was supported by Dalhousie Medical Research Foundation as the Kathryn Weldon Chair of Alzheimer's Research. The authors acknowledge Dr. S. Black, Dr. S. Darvesh, Dr. J. Fisk, Dr. W Chen, Dr. N. Zhang, Dr. R. D'Arcy, Dr, C. Bowen, and Dr. A. Mitnitski, for critical discussions and/or assistance with the development of the Brain Atrophy and Lesion Index approach, Ms. S. Fay with administrative support and with proofreading the final version of the manuscript.

Authors' disclosures available online (http://www.jalz.com/disclosures/view.php?id=2282).

Data collection and sharing for this project was funded by the Alzheimer's Disease Neuroimaging Initiative (ADNI) (National Institutes of Health Grant U01 AG024904) and DOD ADNI (Department of Defense award number W81XWH-12-2-0012). ADNI is funded by the National Institute on Aging, the National Institute of Biomedical Imaging and Bioengineering, and through generous contributions from the following: Alzheimer's Association; Alzheimer's Drug Discovery Foundation; BioClinica, Inc.; Biogen Idec Inc.; Bristol-Myers Squibb Company; Eisai Inc.; Elan Pharmaceuticals, Inc.; Eli Lilly and Company; F. Hoffmann-La Roche Ltd and its affiliated company Genentech, Inc.; GE Healthcare; Innogenetics, N.V.; IXICO Ltd.; Janssen Alzheimer Immunotherapy Research \& Development, LLC.; Johnson \& Johnson Pharmaceutical Research \& Development LLC.; Medpace, Inc.; Merck \& Co., Inc.; Meso Scale Diagnostics, LLC.; NeuroRx Research; Novartis Pharmaceuticals Corporation; Pfizer Inc.; Piramal Imaging; Servier; Synarc Inc.; and Takeda Pharmaceutical Company. The Canadian Institutes of Health Research is providing funds to support ADNI clinical sites in Canada. Private sector contributions are facilitated by the Foundation for the National Institutes of Health (www.fnih.org). The grantee organization is the Northern California Institute for Research and Education, and the study is coordinated by the Alzheimer's Disease Cooperative Study at the University of California, San Diego. ADNI data are disseminated by the Laboratory for Neuro Imaging at the University of Southern California. The authors would also like to acknowledge ADNI database managers for their professional and prompt help with data downloading and sample clarification.

\section{SUPPLEMENTARY MATERIAL}

Supplementary tables are available in the electronic version of this article: http://dx.doi.org/10.3233/JAD140333 .

\section{REFERENCES}

[1] Boyle PA, Wilson RS, Yu L, Barr AM, Honer WG, Schneider JA, Bennett DA (2013) Much of late life cognitive decline is not due to common neurodegenerative pathologies. Ann Neurol 74, 478-489.

[2] van der Vlies AE, Staekenborg SS, Admiraal-Behloul F, Prins ND, Barkhof F, Vrenken H, Reiber JH, Scheltens P, van der Flier WM (2013) Associations between magnetic resonance imaging measures and neuropsychological impairment in early and late onset Alzheimer's disease. J Alzheimers Dis 35, 169-178

[3] Kim KW, MacFall JR, Payne ME (2008) Classification of white matter lesions on magnetic resonance imaging in elderly persons. Biol Psychiatry 64, 273-280.

[4] Vernooij MW, Smits M (2012) Structural neuroimaging in aging and Alzheimer's disease. Neuroimaging Clin N Am 22, 33-55. 
[5] Eckerström C, Olsson E, Klasson N, Bjerke M, Göthlin M, Jonsson M, Rolstad S, Malmgren H, Wallin A, Edman A (2011) High white matter lesion load is associated with hippocampal atrophy in mild cognitive impairment. Dement Geriatr Cogn Disord 31, 132-138.

[6] Muller M, Appelman AP, van der Graaf Y, Vincken KL, Mali WP, Geerlings MI (2011) Brain atrophy and cognition: Interaction with cerebrovascular pathology? Neurobiol Aging 32, 885-893.

[7] Appel J, Potter E, Bhatia N, Shen Q, Zhao W, Greig MT, Raj A, Barker WW, Potter H, Schofield E, Wu Y, Loewenstein DA, Duara R (2009) Association of white matter hyperintensity measurements on brain MR imaging with cognitive status, medial temporal atrophy, and cardiovascular risk factors. AJNR Am J Neuroradiol 30, 1870-1876.

[8] Sinka L, Kövari E, Gold G, Hof PR, Herrmann FR, Bouras C, Giannakopoulos P (2010) Small vascular and Alzheimer disease-related pathologic determinants of dementia in the oldest-old. J Neuropathol Exp Neurol 69, 1247-1255.

[9] Jacobs HI, Clerx L, Gronenschild EH, Aalten P, Verhey FR (2014) White matter hyperintensities are positively associated with cortical thickness in Alzheimer's disease. J Alzheimers Dis 39, 409-422.

[10] Barnes J, Carmichael OT, Leung KK, Schwarz C, Ridgway GR, Bartlett JW, Malone IB, Schott JM, Rossor MN, Biessels GJ, DeCarli C, Fox NC, Alzheimer's Disease Neuroimaging, Initiative (2013) Vascular and Alzheimer's disease markers independently predict brain atrophy rate in Alzheimer's Disease Neuroimaging Initiative controls. Neurobiol Aging 34, 1996-2002.

[11] Chen W, Song X, Zhang Y, Darvesh S, Zhang N, D'Arcy RC, Black S, Rockwood K (2010) An MRI-based semiquantitative index for the evaluation of brain atrophy and lesions in Alzheimer's disease, mild cognitive impairment and normal aging. Dement Geriatr Cogn Disord 30, 121-130.

[12] Zhang N, Song X, Zhang Y, Chen W, D'Arcy RC, Darvesh S, Fisk JD, Rockwood K, Alzheimer's disease Neuroimaging, Initiative (2011) An MRI brain atrophy and lesion index to assess the progression of structural changes in Alzheimer's disease, mild cognitive impairment, and normal aging: A follow-up study. J Alzheimers Dis 26, 359-367.

[13] Fazekas F, Chawluk JB, Alavi A, Hurtig HI, Zimmerman RA (1987) MR signal abnormalities at $1.5 \mathrm{~T}$ in Alzheimer's dementia and normal aging. AJR Am J Roentgenol 149, 351356.

[14] Scheltens P, Barkhof F, Leys D, Pruvo JP, Nauta JJ, Vermersch P, Steinling M, Valk J (1993) A semiquantative rating scale for the assessment of signal hyperintensities on magnetic resonance imaging. J Neurol Sci 114, 7-12.

[15] Scheltens P, Erkinjunti T, Leys D, Wahlund LO, Inzitari D, del Ser T, Pasquier F, Barkhof F, Mäntylä R, Bowler J, Wallin A, Ghika J, Fazekas F, Pantoni L (1998) White matter changes on CT and MRI: An overview of visual rating scales. European Task Force on Age-Related White Matter Changes. Eur Neurol 39, 80-89.

[16] Wahlund LO, Barkhof F, Fazekas F, Bronge L, Augustin M, Sjögren M, Wallin A, Ader H, Leys D, Pantoni L, Pasquier F, Erkinjuntti T, Scheltens P, European Task Force on AgeRelated White Matter, Changes (2001) A new rating scale for age-related white matter changes applicable to MRI and CT. Stroke 32, 1318-1322.

[17] Zhang N, Song X, Zhang Y (2012) Combining structural brain changes improves the prediction of Alzheimer's disease and mild cognitive impairment. Dement Geriatr Cogn Disord 33, 318-326.
[18] Guo H, Song X, Vandorpe R, Zhang Y, Chen W, Zhang N, Schmidt MH, Rockwood K, for the Alzheimer's Disease Neuroimaging, Initiative (2013) Evaluation of common structural brain changes in aging and Alzheimer's disease using an MRI based Brain Atrophy and Lesion Index: A comparison between T1WI and T2WI at 1.5T and 3.0T. AJNR Am $J$ Neuroradiol 35, 504-512.

[19] Song X, Mitnitski A, Zhang N, Chen W, Rockwood K (2013) Dynamics of brain structure and cognitive function in the Alzheimer's disease neuroimaging initiative. J Neurol Neurosurg Psychiatry 84, 71-78.

[20] Weiner MW, Aisen PS, Jack CR Jr, Jagust WJ, Trojanowski JQ, Shaw L, Saykin AJ, Morris JC, Cairns N, Beckett LA, Toga A, Green R, Walter S, Soares H, Snyder P, Siemers E, Potter W, Cole PE, Schmidt M, Alzheimer's Disease Neuroimaging, Initiative (2010) The Alzheimer's Disease Neuroimaging Initiative: Progress report and future plans. Alzheimers Dement 6, 201-211.

[21] Jack CR Jr, Bernstein MA, Fox NC, Thompson P, Alexander G, Harvey D, Borowski B, Britson PJ, Whitwell L, Ward J, Dale C, Felmlee AM, Gunter JP, Hill JL, Killiany DL, Schuff R, Fox-Bosetti N, Lin S, Studholme C, DeCarli C, Krueger CS, Ward G, Metzger HA, Scott GJ, Mallozzi KT, Blezek R, Levy D, Debbins J, Fleisher JP, Albert AS, Green M, Bartzokis R, Glover G, Mugler G, Weiner J, MW (2008) The Alzheimer's Disease Neuroimaging Initiative (ADNI): MRI methods. J Magn Reson Imaging 27, 685-691.

[22] Aisen PS, Petersen RC, Donohue MC, Gamst A, Raman R, Thomas RG, Walter S, Trojanowski JQ, Shaw LM, Beckett LA, Jack CR Jr, Jagust W, Toga AW, Saykin AJ, Morris JC, Green RC, Weiner MW, Alzheimer's Disease Neuroimaging, Initiative (2010) Clinical core of the Alzheimer's Disease Neuroimaging Initiative: Progress and plans. Alzheimers Dement 6, 239-246.

[23] McKhann G, Drachman D, Folstein M, Katzman R, Price D, Stadlan EM (1984) Clinical diagnosis of Alzheimer's disease: Report of the NINCDS-ADRDA Work Group under the auspices of Department of Health and Human Services Task Force on Alzheimer's Disease. Neurology 34, 939-944.

[24] Dubois B, Feldman HH, Jacova C, Dekosky ST, BarbergerGateau P, Cummings J, Delacourte A, Galasko D, Gauthier S, Jicha G, Meguro K, O’brien J, Pasquier F, Robert P, Rossor M, Salloway S, Stern Y, Visser PJ, Scheltens P (2007) Research criteria for the diagnosis of Alzheimer's disease: Revising the NINCDS-ADRDA criteria. Lancet Neurol 6, 734-746.

[25] Shaw LM, Vanderstichele H, Knapik-Czajka M, Clark CM, Aisen PS, Petersen RC, Blennow K, Soares H, Simon A, Lewczuk P, Dean R, Siemers E, Potter W, Lee VM, Trojanowski JQ, Alzheimer's Disease Neuroimaging, Initiative (2009) Cerebrospinal fluid biomarker signature in Alzheimer's disease neuroimaging initiative subjects. Ann Neurol 65, 403-413.

[26] Kwee RM, Kwee TC (2007) Virchow-Robin spaces at MR imaging. Radiographics 27, 1071-1086.

[27] Chen W, Song X, Zhang Y (2011) Assessment of the Virchow-Robin spaces in Alzheimer's disease, mild cognitive impairment, and normal aging, using high-field MRI. AJNR Am J Neuroradiol 32, 1490-1495.

[28] Patankara TF, Mitra D, Varma A, Snowden J, Neary D, Jackson A (2005) Dilatation of the Virchow-Robin Space is a sensitive indicator of cerebral microvascular disease: Study in elderly patients with dementia. AJNR Am J Neuroradiol 26, 1512-1520.

[29] Marín-Padilla M1, Knopman DS (2011) Developmental aspects of the intracerebral microvasculature and perivascu- 
lar spaces: Insights into brain response to late-life diseases. J Neuropathol Exp Neurol 70, 1060-1069.

[30] Jagust WJ, Zheng L, Harvey DJ, Mack WJ, Vinters HV, Weiner MW, Ellis WG, Zarow C, Mungas D, Reed BR, Kramer JH, Schuff N, DeCarli C, Chui HC (2008) Neuropathological basis of magnetic resonance images in Aging and Dementia. Ann Neurol 63, 72-80.

[31] Bryan RN, Cai J, Burke G, Hutchinson RG, Liao D, Toole JF, Dagher AP, Cooper L (1999) Prevalence and anatomic characteristics of infarct-like lesions on MR images of middleaged adults: The atherosclerosis risk in communities study. AJNR Am J Neuroradiol 20, 1273-1280.

[32] Duvernoy HM (1999) The Human Brain: Surface, ThreeDimensional Sectional Anatomy with MRI, and Blood Supply, 2nd edition, Springer, New York, pp.

[33] Chavhan, Jankharia GB, Jankharia B (2012) Cross Sectional Anatomy $C T \& M R I$, Jaypee, London.

[34] Gouw AA, van der Flier WM, Pantoni L, Inzitari D, Erkinjuntti T, Wahlund LO, Waldemar G, Schmidt R, Fazekas F, Scheltens P, Barkhof F, study LADIS, group (2008) On the etiology of incident brain lacunes: Longitudinal observations from the LADIS study. Stroke 39, 3083-3085.

[35] Staekenborg SS, Koedam EL, Henneman WJ, Stokman P, Barkhof F, Scheltens P, van der Flier WM (2009) Progression of mild cognitive impairment to dementia: Contribution of cerebrovascular disease compared with medial temporal lobe atrophy. Stroke 40, 1269-1274.

[36] Defrancesco M, Marksteiner J, Deisenhammer E, Kemmler G, Djurdjevic T, Schocke M (2013) Impact of white matter lesions and cognitive deficits on conversion from mild cognitive impairment to Alzheimer's disease. J Alzheimers Dis 34, 665-672.

[37] Kim HJ, Kang SJ, Kim C, Kim GH, Jeon S, Lee JM, Oh SJ, Kim JS, Choe YS, Lee KH, Noh Y, Cho H, Yoon CW, Chin J, Cummings JL, Lee JH, Na DL, Seo SW (2013) The effects of small vessel disease and amyloid burden on neuropsychiatric symptoms: A study among patients with subcortical vascular cognitive impairments. Neurobiol Aging 34, 19131920.

[38] Frisoni GB, Fox NC, Jack CR Jr, Scheltens P, Thompson PM (2010) The clinical use of structural MRI in Alzheimer disease. Nat Rev Neurol 6, 67-77.

[39] Knopman DS, DeKosky ST, Cummings JL, Chui H, CoreyBloom J, Relkin N, Small GW, Miller B, Stevens JC (2001) Practice parameter: Diagnosis of dementia (an evidencebased review): Report of the Quality Standards Subcommittee of the American Academy of Neurology. Neurology 56, 11431153.

[40] Gouw AA, Van der Flier WM, van Straaten EC, Barkhof F, Ferro JM, Baezner H, Pantoni L, Inzitari D, Erkinjuntti T, Wahlund LO, Waldemar G, Schmidt R, Fazekas F, Scheltens P, Study LADIS, Group (2006) Simple versus complex assessment of white matter hyperintensities in relation to physical performance and cognition: The LADIS study. J Neurol 253, 1189-1196.

[41] Kuczynski B, Targan E, Madison C, Weiner M, Zhang Y, Reed B, Chui HC, Jagust W (2010) White matter integrity and cortical metabolic associations in aging and dementia. Alzheimers Dement 6, 54-62.

[42] Black S, Gao F, Bilbao J (2009) Understanding white matter disease: Imaging - pathological correlations in vascular cognitive impairment. Stroke 40, S48-S52.

[43] Jack CR Jr (2012) Alzheimer disease: New concepts on its neurobiology and the clinical role imaging will play. Radiology 263, 344-361.

[44] Savva GM, Wharton SB, Ince PG, Forster G, Matthews FE, Brayne C, Medical Research Council Cognitive Function, Ageing, Study (2009) Age, neuropathology, and dementia. $N$ Engl J Med 360, 2302-2309.

[45] Gouw AA, Seewann A, van der Flier WM, Barkhof F, Rozemuller AM, Scheltens P, Geurts JJ (2011) Heterogeneity of small vessel disease: A systematic review of MRI and histopathology correlations. J Neurol Neurosurg Psychiatry 82, 126-135 